\title{
Glukose-Echtzeitmessung wird Kassenleistung
}

\begin{abstract}
Für Typ-1-Diabetiker mit intensivierter Insulintherapie wird die kontinuierliche Glukosemessung Kassenleistung. „Ein Segen für die Patienten", so die Deutsche Diabetes Gesellschaft (DDG).
\end{abstract}

Bei dem Continuous Glucose Monitoring (CGM) mit Real-Time-Messgeräten wird mit einem fadenförmigen Sensor permanent der Glukosegehalt in der interstitiellen Flüssigkeit des Unterhautfettgewebes gemessen. Die Werte werden an ein tragbares Empfangsgerät gesendet. Auf dem Display des Empfangsgerätes kann der Diabetiker jederzeit den aktuellen

\section{DATEN UND FAKTEN}

\section{$75 \%$}

der Diabetiker sterben an Gefäßerkrankungen, schätzt die Deutsche Diabetes Gesellschaft (DDG). Häufigste Todesursache ist dabei der Herzinfarkt, gefolgt vom Schlaganfall. Bereits bei der Diagnosestellung von Typ-2-Diabetes sind oft schon makrovaskuläre Veränderungen vorhanden. Typ-2-Diabetiker entwickeln zudem häufig eine Herzinsuffizienz, die rechtzeitig diagnostiziert werden muss. Die Prognose Betroffener ist äußerst ungünstig. Auf die erniedrigte Nierenfunktion ist zu achten.

(eis)

\section{INFOS IM INTERNET}

Alle Folgen der Sommer-Akademie finden Sie im Web unter www.aerztezeitung.de

Weitere Informationen zum Thema Diabetes gibt es unter www.springermedizin.de unter "Diabetes" in der Suchmaske!

Unter www.deutsche-diabetes-gesellschaft. de finden Sie Infos aus der Forschung, zur Behandlung und zur Weiterbildung, unter www. diabetesde.org zudem Patienteninfos.

Berichte zu sozial- und berufspolitischen Themen von Diabetologen gibt es beim Bundesverband niedergelassener Diabetologen unter der Adresse www.bvnd.de
Glukosewert sowie den Trend ablesen und entsprechend durch Nahrungsaufnahme oder Insulingabe einer Stoffwechselentgleisung entgegensteuern.

„Hierdurch lassen sich die Blutzuckerselbstmessungen verringern und die Stoffwechsellage langfristig verbessern, ohne dass dabei das Risiko schwerer Unterzuckerungen in Kauf genommen werden muss", begründet der Gemeinsame Bundesausschuss (G-BA) die Aufnahme der CGM in den Leistungskatalog. Zur kontinuierlichen Messung wird besonders dann geraten, wenn die Therapieziele ohne CGM nicht erreicht werden können.

\section{Voraussetzung für die Verordnung}

Die Verordnung der CGM ist an bestimmte Qualifikationen gebunden. Vornehmen dürfen sie nur Fachärzte für Innere Medizin und Endokrinologie und Diabetologie, außerdem Internisten, Allgemeinmediziner und Pädiater mit der zusätzlichen Anerkennung „Diabetologie“ oder „Diabetologe Deutsche Diabetes Gesellschaft". Die einsetzbaren Messgeräte müssen als CGM zugelassen sein und über eine Alarmfunktion mit individuell einstellbaren Glukosegrenzwerten verfügen. Werden beim Einsatz des Gerätes personenbezogene Daten verwendet, muss sichergestellt sein, dass hierauf kein Zugriff durch Dritte möglich ist.

Die Systeme liefern kontinuierlich umfassende Informationen über den Glukoseverlauf. Mit dieser Menge an Daten muss ein Diabetespatient erst einmal umgehen können. Und vollständig verzichten können Anwender auf die Blutglukosemessung nicht. Die Arbeitsgemeinschaft Diabetes \& Technologie (AGDT) der DDG rät Patienten, immer ein Blutzucker(BZ)-Messsystem mit sich zu führen, um bei Ausfall des CGM gerüstet zu sein.

Dabei muss beachtet werden, dass der Gewebezuckerwert nicht mit dem BZWert gleichzusetzen ist. Schwankt z.B. der BZ stark, etwa beim Sport, wird sich der Gewebezucker nur mit Verzögerung entsprechend ändern. Die Unterschiede

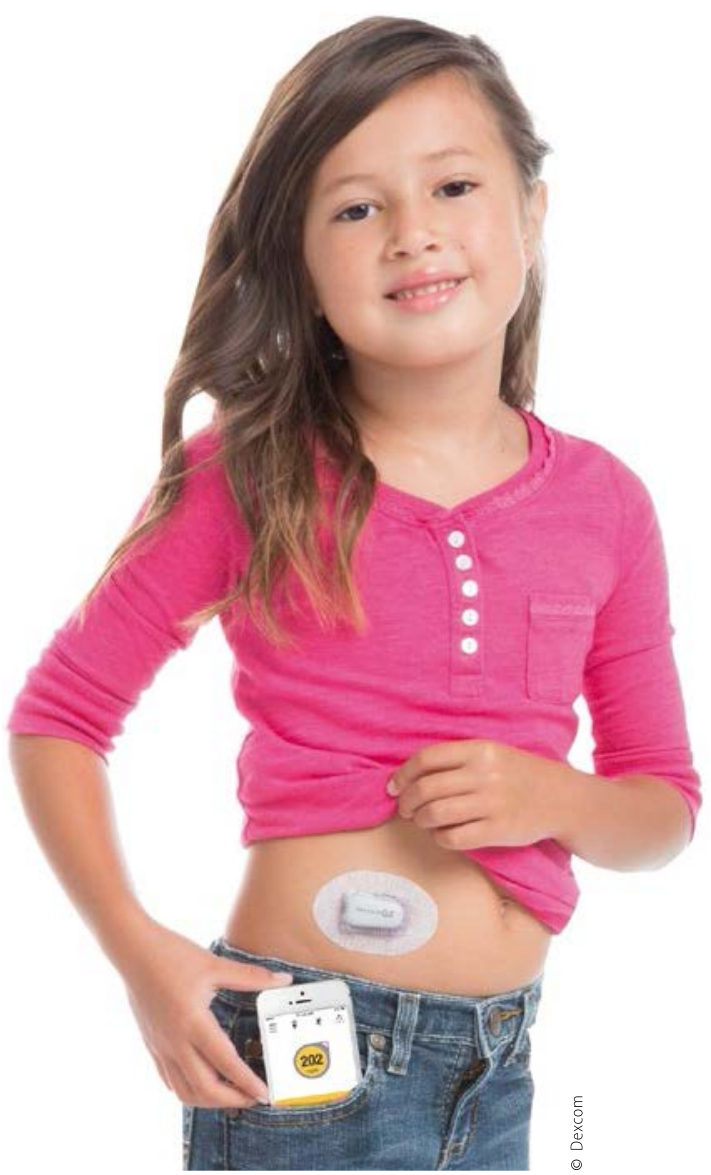

System zur kontinuierlichen Glukosemessung: Ein Sensor misst die Zuckerwerte im Unterhautfettgewebe und sendet sie an ein tragbares Empfangsgerät.

zwischen den Kompartimenten interstitielle Flüssigkeit und Blut könnten in Phasen schneller Glukoseänderungen erheblich sein, so die AGDT. „Bisher besteht keine allgemein akzeptierte evidenzbasierte Empfehlung, wie diese Unterschiede in sichere, adäquat adjustierte Therapieentscheidungen umgewandelt werden sollen."

\section{Adäquate Schulung ist essenziell}

Es bedarf also einiger Erfahrung mit der Gewebezuckermessung, bevor Patienten auf dieser Grundlage Anpassungen ihrer Insulintherapie vornehmen können. Im Zweifelsfall soll immer per BZ-Messung kontrolliert werden.

Damit ist klar: Die Grundprinzipien der Diabetestherapie müssen verstanden sein und eine adäquate Schulung ist essenziell, ebenso wie die Adhärenz der Patienten. Denn die CGM-Geräte müssen regelmäßig kalibriert werden. Dennoch: Es ist im Alltag praktikabler, sich den Sensor $\mathrm{zu}$ setzen, ab und $\mathrm{zu} \mathrm{zu}$ kalibrieren und dafür tagsüber auf das BZ-Messgerät verzichten zu können, als pro Tag sechs oder sieben BZ-Werte zu messen. (ner/eis) 